Pacific Journal of Mathematics

SUR LES SUITES D'INTERPOLATION EN PLUSIEURS 


\title{
SUR LES SUITES D'INTERPOLATION EN PLUSIEURS VARIABLES
}

\author{
Denise et Eric Amar
}

\begin{abstract}
We prove that there exists a sequence $\sigma$ in the polydisc $D^{2}$ of $\mathrm{C}^{2}$ (resp in the unit ball $\mathrm{B}_{2}$ of $\mathrm{C}^{2}$ ) such that $\sigma$ is strongly $H^{2}\left(D^{2}\right)$ (resp. $H^{2}\left(\mathbf{B}^{2}\right)$ ) interpolating but not $H^{\infty}\left(\mathbf{D}^{2}\right)$ (resp. $H^{\infty}\left(\mathbf{B}_{2}\right)$ ) interpolating. These results are corollaries of a result of this kind for the Bergman class $A^{2}(D)$ of the unit disc.
\end{abstract}

1. Introduction et notations. Soit $\lambda$ la mesure de Lebesgue normalisée sur le disque unité $\mathbf{D}$ de $\mathbf{C}$; on note $H^{\infty}(\mathbf{D})$ l'algèbre des fonctions analytiques et bornées dans $\mathbf{D}$ et $A^{2}(\mathbf{D})$ l'espace de Bergman des fonctions analytiques dans $\mathbf{D}$ telles que:

$$
\int_{\mathbf{D}}|f(z)|^{2} d \lambda(z)=\|f\|_{2}^{2}<+\infty \text {. }
$$

Soit $\sigma=\left\{z_{n}, n \in \mathbf{N}\right\}$ une suite dans $\mathbf{D}$, on dit que $\sigma$ est fortement d'interpolation $A^{2}(\mathbf{D})$ si l'opérateur $T_{2}$ défini sur $A^{2}(\mathbf{D})$ dans l'espace des suites par

$$
\forall f \in A^{2}(\mathbf{D}), \quad T_{2} f=\left\{\left(1-\left|z_{n}\right|^{2}\right) f\left(z_{n}\right), \quad n \in \mathbf{N}\right\}
$$

est continu et surjectif sur $l^{2}(\mathbf{N})$;

on dit que $\sigma$ est d'interpolation $H^{\infty}(\mathbf{D})$ si l'opérateur $T_{\infty}$ de $H^{\infty}(\mathbf{D})$ dans l'espace des suites définit par:

$$
\forall f \in H^{\infty}(\mathbf{D}), \quad T_{\infty} f=\left\{f\left(z_{n}\right), n \in \mathbf{N}\right\}
$$

est surjectif sur $l^{\infty}(\mathbf{N})$.

Dans cette note on montre le

THEOREME 1. Il existe une suite $\sigma$ qui est fortement d'interpolation $A^{2}(\mathbf{D})$ mais qui n'est pas d'interpolation $H^{\infty}(\mathbf{D})$.

Ce résultat contraste avec la cas de la classe de $H a r d y H^{2}(\mathbf{D})$ car on sait [6] que interpolation $H^{2}(\mathbf{D})$ entraîne interpolation $H^{\infty}(\mathbf{D})$.

Soit $d \mu$ la mesure de Lebesgue normalisée sur le tore $\mathbf{T}^{2}=$ $\left\{(z, w) \in \mathbf{C}^{2},|z|=|w|=1\right\}$ (respectivement $d \mu$ la mesure de Lebesgue 
normalisée sur la sphère $\left.S_{2}=\partial \mathbf{B}_{2}=\left\{(z, w) \in \mathbf{C}^{2},|z|^{2}+|\boldsymbol{w}|^{2}=1\right\}\right)$ on note encore $H^{\infty}\left(\mathbf{D}^{2}\right)$ l'algèbre des fonctions analytiques et bornées dans le polydisque $\mathbf{D}^{2}$ et $H^{2}\left(\mathbf{S}^{2}\right)$ l'espace des fonctions analytiques $f$ dans $\mathbf{D}^{2}$ telles que:

$$
\sup _{r<1} \int_{\mathbf{T}^{2}}|f(r z, r w)|^{2} d m(z, w)=\|f\|_{2}^{2}<+\infty
$$

De même $H^{\infty}\left(\mathbf{B}_{2}\right)$ désignera l'algèbre des fonctions analytiques et bornées dans $\mathbf{B}_{2}=\left\{(z, w) \in \mathbf{C}^{2} ;|z|^{2}+|w|^{2}<1\right\}$ et $H^{2}\left(\mathbf{B}_{2}\right)$ l'espace des fonctions analytiques $f$ dans $\mathbf{B}_{2}$ telles que

$$
\sup _{r<1} \int_{S_{2}}|f(r z, r w)|^{2} d \mu(z, w)=\|f\|_{2}^{2}<+\infty
$$

Soit $\sigma=\left(\underline{z}_{n}, n \in \mathbf{N}\right)$ une suite de $\mathbf{D}^{2}$ (resp. de $\mathbf{B}_{2}$ ) on dit que $\sigma$ est d'interpolation $H^{\infty}\left(\mathbf{D}^{2}\right) \quad$ si l'opérateur $T_{\infty}: \forall f \in H^{\infty}\left(\mathbf{D}^{2}\right), \quad T_{\infty} f=$ $\left\{f\left(\underline{z}_{n}\right), n \in \mathbf{N}\right\}$ est surjectif sur $l^{\infty}(\mathbf{N})$ (de même pour $H^{\infty}\left(\mathbf{B}_{2}\right)$ ).

On dit que $\sigma$ est fortement d'interpolation $H^{2}\left(\mathbf{D}^{2}\right)$ si l'opérateur

$$
T_{2}: \forall f \in H^{2}\left(\mathbf{D}^{2}\right), \quad T_{2} f=\left\{\left(1-\left|z_{n}\right|^{2}\right)^{\frac{1}{2}}\left(1-\left|w_{n}\right|^{2}\right)^{\frac{1}{2}} f\left(\underline{z}_{n}\right), n \in \mathbf{N}\right\}
$$

est surjectif et continu sur $l^{2}(\mathbf{N})$, avec $\underline{z}_{n}=\left(z_{n}, w_{n}\right)$.

De même $\sigma$ est fortement d'interpolation pour $H^{2}\left(\mathbf{B}_{2}\right)$ si

$$
T_{2}: \forall f \in H^{2}\left(\mathbf{B}_{2}\right), T_{2} f=\left\{\left(1-\left|\underline{z}_{n}\right|^{2}\right) f\left(\underline{z}_{n}\right), n \in \mathbf{N}\right\}
$$

est surjectif et continu sur $l^{2}(\mathbf{N}),\left(\underline{z}_{n}=\left(z_{n}, w_{n}\right),\left|\underline{z}_{n}\right|^{2}=\left|z_{n}\right|^{2}+\left|w_{n}\right|^{2}\right)$.

Comme application du théorème 1 , on montre alors:

THEOREME 2. Il existe une suite $\sigma$ dans $\mathbf{D}^{2}$ qui est fortement d'interpolation $H^{2}\left(\mathbf{D}^{2}\right)$ mais $n$ 'est pas d'interpolation $H^{\infty}\left(\mathbf{D}^{2}\right)$.

THeoreme 3. Il existe une suite $\sigma$ dans $\mathbf{B}_{2}$ qui est fortement d'interpolation $\mathrm{H}^{2}\left(\mathbf{B}_{2}\right)$ mais n'est pas d'interpolation $\mathrm{H}^{\infty}\left(\mathbf{B}_{2}\right)$.

Une autre application du théorème 1 montre que le classique théorème de Pick-Nevanlinna [4] [5] ne se généralise pas du tout en plusieurs variables [1].

2. Preuve du Théorème 1 . On note $K_{z}(\zeta)$ le noyau de Cauchy-Bergman de $\mathbf{D}$ et $E_{z}(\zeta)$ ce noyau normalisé dans $A^{2}(\mathbf{D})$ : 


$$
E_{z}(\zeta)=\frac{K_{z}(\zeta)}{\left\|K_{z}\right\|}=\frac{1-|z|^{2}}{(1-\bar{z} \zeta)^{2}}
$$

Dans [2], on montre que la suite $\left(z_{n}\right)_{n \in \mathrm{N}}\left(z_{n}\right) \subset D$ est d'interpolation pour $A^{2}(\mathbf{D})$ si et seulement si l'opérateur $S$ de $l^{2}(\mathbf{N})$ défini par la matrice $\left(\left\langle E_{z n}, E_{z k}\right\rangle\right)_{n, k}$ est bicontinu. Une suite d'interpolation de $H^{\infty}(D)$ est une suite d'interpolation pour $A^{2}(\mathbf{D})$ [2].

(b) Construction d'une suite d'interpolation de $A^{2}(\mathbf{D})$ qui n'est pas d'interpolation pour $H^{\infty}(\mathbf{D})$.

Cette suite $(\sigma)$ sera réunion de suites finies $G_{n}$ de points situés sur unmême cercle et équiréparties sur ce cercle

$$
G_{n}=\left\{z_{k} ;\left|z_{k}\right|=1-2^{-g(n)}, \quad \operatorname{Arg} z_{k}=\frac{2 k \pi}{2^{g(n)}} \quad 0 \leqq k<g(n)\right\}
$$

où $g$ est une fonction strictement croissante. $G_{n}$ est la $n^{\text {ième }}$ génération de la suite $\sigma$ au sens de Garnett [3]. $\sigma=\cup_{n} G_{n} n$ 'est pas une suite d'interpolation de $H^{\infty}(D)$. En effet $\sum_{z_{k} \in G_{n}}\left(1-\left|z_{k}\right|\right)=1$ d'où la suite $\sum_{z_{i} \in \sigma}\left(1-\left|z_{i}\right|\right)$ est divergente. Par construction, chaque génération est un ensemble d'interpolation de $A^{2}(\mathbf{D})$ pour une même constante $C$. On montre qu'on peut choisir la fonction $g$ pour que $\sigma$ soit d'interpolation pour $A^{2}(\mathbf{D})$.

DÉmonstration. Soit $S_{p}$ la matrice $\left(\left\langle E_{z}, E_{z_{j}}\right\rangle\right)_{i j}, z_{i} \in G_{p}, z_{j} \in G_{p} . S_{p}$ est bicontinue et

$$
\begin{aligned}
& \left\|S_{p}\right\| \leqq C^{2} \\
& \left\|S_{p}^{-1}\right\| \leqq C^{2} .
\end{aligned}
$$

On note $T_{n}$ la matrice $\left(\left\langle E_{z_{i}}, E_{z_{j}}\right\rangle\right)_{i j}, \quad z_{i} \in \cup_{1}^{n} G_{p}, \quad z_{j} \in \cup_{1}^{n} G_{p}$. On démontre par récurrence que $T_{n}$ est une matrice inversible. Supposons

$$
\begin{aligned}
& \left\|T_{n}\right\| \leqq K_{n}^{2} \quad \text { où } \quad C^{2} \leqq K_{n}^{2} \\
& \left\|T_{n}^{-1}\right\| \leqq K_{n}^{2} .
\end{aligned}
$$

$T_{n+1}$ est de la forme

$$
\left(\begin{array}{cc}
T_{n} & G \\
G^{*} & S_{n+1}
\end{array}\right)
$$

où $G$ est la matrice $\left(\left\langle E_{z_{k}}, E_{z_{p}}\right\rangle, z_{k} \in \cup_{1}^{n} G_{j}, z_{p} \in G_{n+1}\right)$. Si $z_{k} \in G_{j}$, $z_{p} \in G_{n+1}$ 


$$
\begin{aligned}
&\left|\left\langle E_{z_{k}}, E_{z_{p}}\right\rangle\right|=\frac{\left(1-\left|z_{k}\right|^{2}\right)\left(1-\left|z_{p}\right|^{2}\right)}{\left|1-\bar{z}_{k} z_{p}\right|^{2}} \\
& \leqq \frac{42^{-g(j)-g(n+1)}}{\left[1-\left(1-2^{-g(j)}\right)\left(1-2^{-g(n+1)}\right]^{2}\right.} \\
& \leqq 42^{g(j)-g(n+1)} \\
&\left|\left\langle E_{z_{k}}, E_{z_{p}}\right\rangle\right|^{2} \leqq 162^{2 g(j)-2 g(n+1)} \\
& \sum_{z_{k} \in G_{j}, z_{p} \in G_{n+1}}\left|\left\langle E_{z_{k}}, E_{z_{p}}\right\rangle\right|^{2} \leqq 16.2^{3 g(j)-g(n+1)}
\end{aligned}
$$

d'où

$$
\begin{aligned}
\sum_{z_{k} \in \cup_{1}^{n} G_{j}, z_{p} \in G_{n+1}}\left|\left\langle E_{z_{k}}, E_{z_{p}}\right\rangle\right|^{2} & \leqq 16.2^{-g(n+1)}\left(\sum_{1}^{n} 2^{3 g(j)}\right) \\
& =\epsilon^{2}(n) .
\end{aligned}
$$

La fonction $g$ sera choisie telle que:

$$
\sum_{n} \epsilon(n)<\frac{1}{2 C^{2}}
$$

La norme de Hilbert-Schmidt de la matrice $G$ est inférieure à $\epsilon(n)$. Si $\lambda \in l^{2}\left(2^{g(1)}+2^{g(2)}+\cdots+2^{g(n)}+2^{g(n+1)}\right)$ alors $\lambda=\mu+\nu$ oú:

$$
\begin{gathered}
\mu \in l^{2}\left(2^{g(1)}+\cdots+2^{g(n)}\right), \nu \in l^{2}\left(2^{g(n+1)}\right) \\
\|\lambda\|^{2}=\|\mu\|^{2}+\|\nu\|^{2} \\
\left\|T_{n+1} \lambda\right\|^{2}=\left\|T_{n} \mu+G \nu\right\|^{2}+\left\|G^{\infty} \mu+S_{n+1} \nu\right\|^{2} \\
\left\|T_{n+1} \lambda\right\|^{2} \leqq\left(K_{n}^{2}\|\mu\|+\epsilon(n)\|\nu\|\right)^{2}+\left(C^{2}\|\nu\|+\epsilon(n)\|\mu\|\right)^{2} \\
\leqq\left[K_{n}^{2}+\epsilon(n)\right]^{2}\|\lambda\|^{2}
\end{gathered}
$$

de même

$$
\left\|T_{n+1}(\lambda)\right\|^{2} \geqq\left[\frac{1}{K_{n}^{2}}-\epsilon(n)\right]^{2}\|\lambda\|^{2}
$$

La matrice $T_{n+1}$ de $\bigcup_{1}^{n+1} G_{p}$ vérifie donc

$$
\begin{aligned}
& \left\|T_{n+1}\right\| \leqq K_{n}^{2}+\epsilon(n) \\
& \left\|T_{n+1}^{-1}\right\| \leqq \frac{1}{\frac{1}{K_{n}^{2}}-\epsilon(n)}
\end{aligned}
$$


La matrice $S$ de $\cup_{n} G_{n}$ vérifiera donc

$$
\begin{aligned}
& \|S\| \leqq C^{2}+\sum_{n} \epsilon(n) \leqq 2 C^{2} \\
& \left\|S^{-1}\right\| \leqq \frac{1}{\frac{1}{C^{2}}-\sum_{n} \epsilon(n)} \leqq 2 C^{2}
\end{aligned}
$$

d'où $\cup_{n} G_{n}$ est une suite d'interpolation pour $A^{2}(\mathbf{D})$.

\section{Applications.}

Preuve du Théorème 2. On considère la suite $\tilde{\boldsymbol{\sigma}}=\left\{\left(z_{n}, z_{n}\right), n \in \mathbf{N}\right\}$ où $\sigma=\left\{z_{n}, n \in \mathbf{N}\right\}$ est la suite construite dans la preuve du Théorème 1; clairement, puisque $\sigma$ n'est pas d'interpolation $H^{\infty}(\mathbf{D}), \tilde{\sigma}$ n'est pas d'interpolation $H^{\infty}\left(\mathbf{D}^{2}\right)$.

Si l'on note

$$
e_{(z, w)}(\xi, \eta)=\frac{\left(1-|z|^{2}\right)^{1 / 2}\left(1-|w|^{2}\right)^{1 / 2}}{(1-\bar{z} \xi)(1-\bar{w} \eta)}
$$

le noyau de Cauchy Szegö $\mathbf{D}^{2}$ normalisé dans $H^{2}\left(\mathbf{D}^{2}\right)$ on a facilement

$$
\forall n, p \in \mathbf{N},\left\langle e_{\left(z_{n}, z_{n}\right)}, e_{\left(z_{p}, z_{p}\right)}\right\rangle=\left\langle E_{z_{n}}, E_{z_{p}}\right\rangle
$$

donc les matrices $\left(\left\langle e_{\left(z_{n}, z_{n}\right)}, e_{\left(z_{p}, z_{p}\right)}\right\rangle,(n, p) \in \mathbf{N}^{2}\right)$ et $\left(\left\langle E_{z_{n}}, E_{z_{p}}\right\rangle,(n, p) \in \mathbf{N}^{2}\right)$ définissent le même opérateur sur $l^{2}(\mathbf{N})$.

On en déduit alors aisément que $\sigma$ est fortement d'interpolation $H^{2}\left(\mathbf{D}^{2}\right)$.

Preuve $d u$ Théorème 3 . On considère dans $\mathbf{B}_{2}$ la suite $\tilde{\tilde{\sigma}}=$ $\left\{\left(z_{n}, 0\right), n \in \mathbf{N}\right\}$ et on remarque, comme ci-dessus que $\left\langle e_{\left(z_{n}, 0\right)}, e_{\left(z_{p}, 0\right)}\right\rangle=$ $\left\langle E_{z_{n}}, E_{z_{p}}\right\rangle$ où ici $e_{\left(z_{n}, 0\right)}$ désigne le noyau de Cauchy-Szegö normalisé dans $H^{2}\left(\mathbf{B}_{2}\right)$.

\section{REFERENCES}

1. D. et E. Amar, Sur les théorèmes de Schwarz-Pick et Nevanlinna dans $C^{n}$, Preprint, Anal. Harm. Orsay, 167 (1975).

2. E. Amar, Méthodes hilbertiennes et interpolation dans le spectre d'une algèbre de Banach, Preprint Anal. Harm. Orsay, 152 (1975) et thèse (1977).

3. J. Garnett, Interpolating sequences for bounded harmonic functions. Indiana Univ. Math. J., 21 (1971). 
4. R. Nevanlinna, Über beschränkte Funktionen die in gegebenen Punkten vorgeschnebene Werte aunehmen, Ann. Acad. Sci. Fenn. ser. A, 13 (1919), n. 1.

5. G. Pick, Über die Beschränkungen analystischer Funktionen, welche durch vergegebene Funktionswerke bewirkt werden, Math. Ann., 77 (1961), 7-23.

6. H. Shapiro, et A. L. Shields, On some interpolation problems for analytic functions, Amer. Math. Soc. Transl., 83 (1961).

Received January 28, 1976 and in revised form May 3, 1977.

UNIVERSITE PARIS XI

91405 ORSAY, FRANCE 


\section{PACIFIC JOURNAL OF MATHEMATICS EDITORS}

ICHARD ARENS (Managing Editor)

niversity of California

os Angeles, CA 90024

\section{. A. BEAUmont}

niversity of Washington

sattle, WA 98105

. C. MOORE

niversity of California

erkeley, CA 94720
J. DUGUNDJI

Department of Mathematics

University of Southern California

Los Angeles, CA 90007

R. FINN AND J. MiLgRAM

Stanford University

Stanford, CA 94305

\section{ASSOCIATE EDITORS}
F. BECKENBACH
B. H. NEUMANN
F. WOLF
K. YosHidA

\section{SUPPORTING INSTITUTIONS}

NIVERSITY OF BRITISH COLUMBIA

UNIVERSITY OF SOUTHERN CALIFORNIA

ALIFORNIA INSTITUTE OF TECHNOLOGY STANFORD UNIVERSITY

NIVERSITY OF CALIFORNIA

ONTANA STATE UNIVERSITY

UNIVERSITY OF HAWAII

NIVERSITY OF NEVADA

UNIVERSITY OF TOKYO

EW MEXICO STATE UNIVERSITY

UNIVERSITY OF UTAH

REGON STATE UNIVERSITY

NIVERSITY OF OREGON

WASHINGTON STATE UNIVERSITY

UNIVERSITY OF WASHINGTON

SAKA UNIVERSITY

AMERICAN MATHEMATICAL SOCIETY

The Supporting Institutions listed above contribute to the cost of publication of this Journal, but they a t owners or publishers and have no responsibility for its contents or policies.

Mathematical papers intended for publication in the Pacific Journal of Mathematics should be in typ Irm or offset-reproduced (not dittoed), double spaced with large margins. Underline Greek letters in re ierman in green, and script in blue. The first paragraph or two must be capable of being used separately as 'nopsis of the entire paper. Items of the bibliography should not be cited there unless absolutely necessary, hich case they must be identified by author and Journal, rather than by item number. Manuscripts, uplicate, may be sent to any one of the four editors. Please classify according to the scheme of Math. Revieu Idex to Vol. 39. All other communications should be addressed to the managing editor, or Elaine Bart Iniversity of California, Los Angeles, California, 90024.

100 reprints are provided free for each article, only if page charges have been substantially pai dditional copies may be obtained at cost in multiples of 50 .

The Pacific Journal of Mathematics is issued monthly as of January 1966. Regular subscription rate: \$72. year (6 Vols., 12 issues). Special rate: $\$ 36.00$ a year to individual members of supporting institutions.

Subscriptions, orders for numbers issued in the last three calendar years, and changes of address should ent to Pacific Journal of Mathematics, 103 Highland Boulevard, Berkeley, California, 94708.

UBLISHED BY PACIFIC JOURNAL OF MATHEMATICS, A NON-PROFIT CORPORATION

Printed at Jerusalem Academic Press, POB 2390, Jerusalem, Israel.

Copyright (C) 1978 Pacific Journal of Mathematics

All Rights Reserved 


\section{Pacific Journal of Mathematics}

\section{Vol. 75, No. $1 \quad$ September, 1978}

Mieczyslaw Altman, General solvability theorems

Denise Amar and Eric Amar, Sur les suites d'interpolation en plusieurs variables ..........................................

Herbert Stanley Bear, Jr. and Gerald Norman Hile, Algebras which satisfy a second order linear partial differential equation ..................

Marilyn Breen, Sets in $R^{d}$ having $(d-2)$-dimensional kernels ............

Gavin Brown and William Moran, Analytic discs in the maximal ideal space

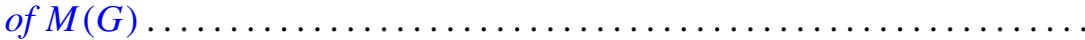

Ronald P. Brown, Quadratic forms with prescribed Stiefel-Whitney

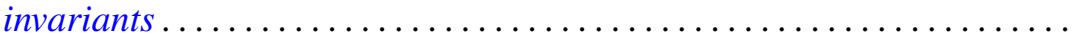

Gulbank D. Chakerian and H. Groemer, On coverings of Euclidean space by convex sets

S. Feigelstock and Z. Schlussel, Principal ideal and Noetherian groups.....

Ralph S. Freese and James Bryant Nation, Projective lattices ............

Harry Gingold, Uniqueness of linear boundary value problems for

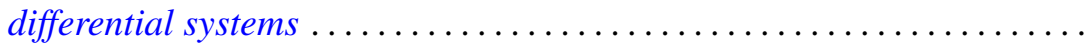

John R. Hedstrom and Evan Green Houston, Jr., Pseudo-valuation domains. . .

William Josephson, Coallocation between lattices with applications to measure extensions

M. Koskela, A characterization of non-negative matrix operators on $l^{p}$ to $l^{q}$ with $\infty>p \geq q>1$

Kurt Kreith and Charles Andrew Swanson, Conjugate points for nonlinear differential equations...........................

Shoji Kyuno, On prime gamma rings ........................ 185

Alois Andreas Lechicki, On bounded and subcontinuous multifunctions ..

Roberto Longo, A simple proof of the existence of modular automorphisms in approximately finite-dimensional von Neumann algebras ...

Kenneth Millett, Obstructions to pseudoisotopy implying isotopy for

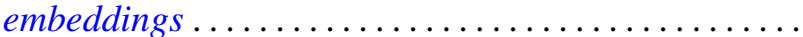

William F. Moss and John Piepenbrink, Positive solutions of elliptic equations. .

Mitsuru Nakai and Leo Sario, Duffin's function and Hadamard's

conjecture

Mohan S. Putcha, Word equations in some geometric semigroups ...

Walter Rudin, Peak-interpolation sets of class $C^{1} \ldots \ldots \ldots$

Elias Saab, On the Radon-Nikodým property in a class of locally convex

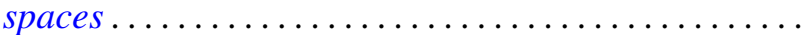

